\title{
Recent experimental results for semileptonic $B$ decays
}

\section{Shigeki Hirose*, for the Belle Collaboration}

Graduate School of Science, Nagoya University

E-mail: shigeki@hepl.phys.nagoya-u.ac.jp

Semileptonic $B$ decays, which contains the $b \rightarrow c$ transition and a leptonic decay of a virtual $W$ boson, provide with an excellent experimental environment for determination of the CabibboKobayashi-Maskawa matrix elements $\left|V_{c b}\right|$ and $\left|V_{u b}\right|$. Besides, specific types of new physics models predict modification of branching fractions and decay topology of semitauonic $B$ decays, which are family of semileptonic decays with the transition $W^{*-} \rightarrow \tau^{-} \bar{v}_{\tau}$. These $B$ decays have been studied by two $B$ Factory experiments: Belle and BaBar, and a hadron-collider experiment LHCb. In this paper, recent experimental results related to semileptonic $B$ decays are discussed.

Flavor Physics and CP Violation,

6-9 June 2016

Caltech, Pasadena CA, USA

${ }^{*}$ Speaker. 


\section{Introduction}

A probability of a $b$ quark to be an $x$ quark $(x=u, c)$ is proportional to the square of the Cabibbo-Kobayashi-Maskawa (CKM) matrix element $\left|V_{x b}\right|$. Since the $W^{*-} \rightarrow \ell^{-} \bar{v}_{\ell}$ part $(l=e, \mu)$ is free from complicated hadronic interaction, semileptonic $B$ decays provide with a clean environment for $\left|V_{x b}\right|$ determination. In addition, due to the massive the $\tau^{-}$lepton, semitauonic $B$ decays such as $\bar{B} \rightarrow D^{(*)} \tau^{-} \bar{v}_{\tau}$ and $\bar{B} \rightarrow \pi^{+} \tau^{-} \bar{v}_{\tau}$ are sensitive to new physics (NP) beyond the Standard Model (SM), for example, the type-II Two Higgs Double Model (2HDM) [1].

In this paper, we discuss the latest experimental results of the semileptonic $B$ decays from the $B$ factory experiments, which have been released in the past year after the European Physics Society meeting 2015.

\section{Detectors and Methods}

Two $B$ Factory experiments were carried out in the 2000's, the one is Belle at the KEKB accelerator and the other is BaBar at the PEP-II accelerator. Both experiments use the $e^{+} e^{-}$colliders with the center-of-mass energy of $10.58 \mathrm{GeV}$, which is precisely consistent with the mass of the $\Upsilon(4 S)$ resonance. Abundant amount of $B$ mesons are produced through the decay of $\Upsilon(4 S) \rightarrow B \bar{B}$ at the high-luminosity accelerators. Belle and BaBar accumulated $772 \times 10^{6}$ and $471 \times 10^{6} B \bar{B}$ pairs, respectively.

In the $\Upsilon(4 S)$ decay, exactly two $B$ mesons are produced without any additional particles. Moreover, the $e^{+} e^{-}$beam energy is precisely known. Therefore the "tagging method" is applicable in the $B$ Factory experiments, where one of two $B$ mesons (tag-side, or $B_{\text {tag }}$ ) is reconstructed from a specific $B$ decay mode so that information of the other $B$ meson of interest (signal-side, or $B_{\text {sig }}$ ) is obtained indirectly.

In the hadronic tagging method, the $B_{\text {tag }}$ meson is reconstructed from its exclusive hadronic decay. As a result of the full-reconstruction of the $B_{\text {tag }}$ meson, a complete four-momentum of the $B_{\text {sig }}$ meson is obtained by taking a difference between the beam momentum and the $B_{\text {tag }}$ momentum. A beam-constrained mass $M_{\mathrm{bc}} \equiv \sqrt{E_{\text {beam }}^{* 2}-\left|\vec{p}_{\text {sig }}^{*}\right|^{2}}$ is used to extract $B_{\text {tag }}$ events, where $E_{\text {beam }}^{*}$ and $\vec{p}_{\text {sig }}^{*}$ denote the beam energy and the $B_{\text {tag }}$ three-momentum, respectively, in the $\Upsilon(4 S)$ rest frame. Due to relatively low branching fractions of each exclusive hadronic $B$ decay mode compared to semileptonic decays, a reconstruction efficiency of $B_{\text {tag }}$ is in the order of $0.1 \%$. Many $B_{\text {tag }}$ decay chains therefore need to be collected to increase the efficiency as much as possible. Belle uses $1149 B_{\text {tag }}$ decay chains in total in a hierarchical multivariate algorithm [2] based on the NeuroBayes package [3]. In BaBar, the semi-inclusive $B_{\text {tag }}$ reconstruction from decay types of $B_{\text {tag }} \rightarrow D_{\text {seed }} Y$ is developed [4], where $D_{\text {seed }}$ refers to a charmed meson $\left(D^{(*) 0}, D^{(*)+}, D_{s}^{(*)+}\right.$ or $J / \psi)$ and $Y$ accounts for up to five charmless meson $\left(\pi^{+}, K^{+}, \pi^{0}\right.$ and $\left.K_{S}^{0}\right)$. The total number of exclusive $B_{\text {tag }}$ decay chains in this method reaches 1768 .

The semileptonic tagging method is firstly developed in the $B^{-} \rightarrow \tau^{-} \bar{v}_{\tau}$ search at BaBar [5] and later applied to the same rare-decay search at Belle [6]. In this method, where a $B_{\text {tag }}$ is reconstructed from exclusive semileptonic decays of $\bar{B}_{\text {tag }} \rightarrow D^{(*)} \ell^{-} \bar{v}_{\ell}$ (sometimes an additional charmless mesons is allowed to exist), full-reconstruction is not possible due to existence of one neutrino in the $B_{\text {tag }}$ decay. In order to obtain $B_{\text {tag }}$ events, kinematic feature of $\cos \theta_{B-D^{(*)} \ell}$ is used, where 
$\theta_{B-D^{(*)} \ell}$ is an angle between momenta of the $B_{\text {tag }}$ and the sum of $D^{(*)}$ and $\ell^{-}$in the $\Upsilon(4 S)$ rest frame. The $\cos \theta_{B-D^{(*)} \ell}$ is calculated by

$$
\cos \theta_{B-D^{(*)} \ell}=\frac{E_{\text {beam }}^{*} E_{D^{(*)} \ell}^{*}-m_{B}^{2}-m_{D^{(*)} \ell}^{2}}{2\left|\vec{p}_{\text {beam }}^{*}\right|\left|\vec{p}_{D^{(*)} \ell}^{*}\right|},
$$

where $E_{D^{(*)} \ell}^{*}$ and $\vec{p}_{D^{(*)} \ell}^{*}$ are a summed energy, and three-momentum of $D^{(*)}$ and $\ell^{-}$. The $m_{B}$ and the $m_{D^{(*)} \ell}$ denote the $B$ meson mass and the invariant mass of the $D^{(*)}-\ell^{-}$system, respectively. All the variables are defined in the $\Upsilon(4 S)$ rest frame. According to Eq. 2.1, it is found that the $\cos \theta_{B-D^{(*)} \ell}$ is a measurable quantity. In order to compose of a closed triangle with the three-momenta of the $B_{\text {tag }}, D^{(*)}-\ell^{-}$system and the unmeasurable neutrino, the value of $\cos \theta_{B-D^{(*)} \ell}$ must be in the region -1 to +1 . Tagging the semileptonic decay done by taking this region of the $\cos \theta_{B-D^{(*)} \ell}$. Although a complete $B_{\text {sig }}$ four-momentum cannot be extracted, this method provide with a relatively larger tagging efficiency at the order of $1 \%$ than that of the hadronic tagging.

\section{Exclusive $\bar{B} \rightarrow X_{c} \ell^{-} \bar{v}_{\ell}$ Measurements}

\section{1 $\left|V_{c b}\right|$ determination with the decay $\bar{B} \rightarrow D \ell^{-} \bar{v}_{\ell}$ at Belle}

It was known that there was a discrepancy by $2-3 \sigma$ between the inclusively-measured and the exclusively-measured CKM matrix element $\left|V_{c b}\right|$. Belle updates the $\left|V_{c b}\right|$ determination by the exclusive decay $\bar{B} \rightarrow D \ell^{-} \bar{v}_{\ell}$ with the full data sample [7], which is about seven times larger statistics than that used in the previous measurement [8]. In this analysis, the hadronic tagging method is applied. After reconstruction of a $B_{\text {tag }}$, a $D$ and a $\ell^{-}$in an event, a missing-mass squared

$$
M_{\text {miss }}^{2}=\left(p_{\text {beam }}-p_{B_{\text {tag }}}-p_{D}-p_{\ell}\right)^{2},
$$

is calculated, where the variable $p$ denotes a four-momentum for a particle specified by the subscript. Signal events clearly peak at $M_{\text {miss }}^{2}=0$ since only one neutrino contributes to $M_{\text {miss }}^{2}$.

The differential decay rate is expressed by

$$
\frac{d \Gamma}{d w}=\frac{G_{F}^{2} m_{D}^{3}}{48 \pi^{3}}\left(m_{B}+m_{D}\right)^{2}\left(w^{2}-1\right)^{\frac{3}{2}} \eta_{\mathrm{EW}}^{2}\left|V_{c b}\right|^{2}|g(w)|^{2},
$$

where $G_{F}$ and $\eta_{\mathrm{EW}}$ denote the Fermi constant and the electroweak correction factor, respectively. The function $g(w)$ is a form factor for hadronic contribution in the $\bar{B} \rightarrow D$ transition. The kinematic variable $w \equiv\left(m_{B}^{2}+m_{D}^{2}-q^{2}\right) /\left(2 m_{B} m_{D}\right)$, where $m_{B}$ and $m_{D}$ are masses of the $B$ meson and the $D$ meson respectively is obtained by measuring a squared transfer momentum $q^{2}$ :

$$
q^{2}=\left(p_{\text {beam }}-p_{B_{\text {tag }}}-p_{D}\right)^{2} .
$$

According to Eq. 3.2, the quantity of $\left|V_{c b}\right|$ is extracted by reconstructing signal events as a function of $w$.

Belle uses two types of the form factor parametrization: CLN form factor, which is a commonlyused parametrization in the exclusive $\left|V_{c b}\right|$ determination based on heavy quark symmetry [9], and BGL form factor, which is more free from model assumption [10]. They yield $\left|V_{c b}\right| \eta_{\mathrm{EW}}=$ 


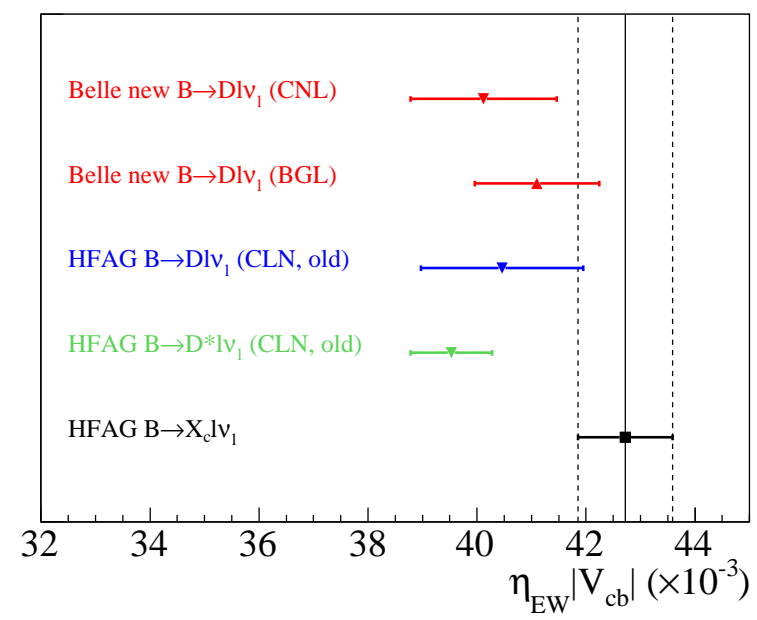

Figure 1: Comparison of $\left|V_{c b}\right|$ with exclusive measurements and the average of inclusive measurements.

$(40.12 \pm 1.34) \times 10^{-3}(\mathrm{CLN})$ and $\left|V_{c b}\right| \eta_{\mathrm{EW}}=(41.10 \pm 1.14) \times 10^{-3}$ (BGL). Figure 1 shows a comparison of these results with the previous measurements. The new Belle result is consistent with both averages of the previous exclusive measurements and the inclusive measurements. Moreover, the result based on the BGL form factor shows a slightly closer value of $\left|V_{c b}\right|$ to the inclusivelymeasured $\left|V_{c b}\right|$ than that based on the CLN form factor.

\section{2 $\bar{B} \rightarrow D^{(*)} \pi^{-} \pi^{+} \ell^{-} \bar{v}_{\ell}$ with hadronic tagging at BaBar}

There was $(1.45 \pm 0.29) \%$ (about $5 \sigma)$ discrepancy between the inclusively-measured and the sum of the exclusively-measured branching fractions of the decay $\bar{B} \rightarrow X_{c} \ell^{-} \bar{v}_{\ell}$ [11], which implies existence of unmeasured exclusive decay modes. BaBar searches for the multi-pion associated decays of $\bar{B} \rightarrow D^{(*)} \pi^{-} \pi^{+} \ell^{-} \bar{v}_{\ell}$ with the hadronic tagging method. The kinamtic variable $U=$ $E_{\text {miss }}-\left|\vec{p}_{\text {miss }}\right|$ is used instead of $M_{\text {miss }}^{2}$, since it is less dependent on modeling of excited $D$ meson states than $M_{\text {miss }}^{2}$.

Table 1 lists signal significances for each decay mode, where cross-feed events from $\bar{B} \rightarrow$ $D^{(*)} \pi^{ \pm} \pi^{0} \ell^{-} \bar{v}_{\ell}$ and $\bar{B} \rightarrow D^{(*)} \pi^{0} \pi^{0} \ell^{-} \bar{v}_{\ell}$ to $\bar{B} \rightarrow D^{(*)} \pi^{+} \pi^{-} \ell^{-} \bar{v}_{\ell}$ modes are treated as signal events. Accordingly, observation of the decay $\bar{B} \rightarrow D^{0} \pi \pi \ell \bar{v}_{\ell}$ and evidence of the decays $\bar{B} \rightarrow D^{(*)+} \pi \pi \ell \bar{v}_{\ell}$ are reported. Based on the isospin symmetry $\mathscr{B}\left(\bar{B} \rightarrow D^{(*)} \pi^{+} \pi^{-} \ell^{-} \bar{v}_{\ell}\right) / \mathscr{B}\left(\bar{B} \rightarrow D^{(*)} \pi \pi \ell^{-} \bar{v}_{\ell}\right)=$ $0.50 \pm 0.17$, the total branching fraction is estimated to be $\mathscr{B}\left(\bar{B} \rightarrow D^{(*)} \pi \pi \ell^{-} \bar{v}_{\ell}\right)=\left(0.52_{-0.07-0.13}^{+0.14+0.27}\right) \%$, where the first uncertainty is the total experimental uncertainty and the second uncertainty arises from the uncertainty of the isospin symmetry assumption described above. The obtained branching fraction fill about one-third of the $1.45 \%$ discrepancy of the inclusive and the exclusive measurements.

\section{Semitauonic $B$ decays}

\section{1 $\bar{B} \rightarrow D^{*} \tau^{-} \bar{v}_{\tau}$ with semileptonic tagging at Belle}

Exclusive semitauonic decays of $\bar{B} \rightarrow D^{(*)} \tau^{-} \bar{v}_{\tau}$ have been measured by the Belle [12, 13, 14], 
Table 1: Significances from the $\bar{B} \rightarrow D^{(*)} \pi^{-} \pi^{+} \ell^{-} \bar{v}_{\ell}$ measurement by BaBar. Systematic uncertainties are considered.

\begin{tabular}{l||c}
\hline \hline Decay mode & Significance \\
\hline $\bar{B} \rightarrow D^{0} \pi \pi \ell \bar{v}_{\ell}$ & $5.0 \sigma$ \\
$\bar{B} \rightarrow D^{+} \pi \pi \ell \bar{v}_{\ell}$ & $3.0 \sigma$ \\
$\bar{B} \rightarrow D^{* 0} \pi \pi \ell \bar{v}_{\ell}$ & $1.6 \sigma$ \\
$\bar{B} \rightarrow D^{*+} \pi \pi \ell \bar{v}_{\ell}$ & $3.0 \sigma$ \\
\hline \hline
\end{tabular}

BaBar [15, 16] and LHCb collaborations [17]. These experiments typically measure the ratio of the branching fractions

$$
R\left(D^{(*)}\right)=\frac{\bar{B} \rightarrow D^{(*)} \tau^{-} \bar{v}_{\tau}}{\bar{B} \rightarrow D^{(*)} \ell^{-} \bar{v}_{\ell}}
$$

in order to cancel common uncertainties such as theoretical uncertainties of form factors, $\left|V_{c b}\right|$ uncertainty and experimental uncertainty of reconstruction efficiencies. The heavy flavor averaging group (HFAG) combined current experimental results of $R(D)$ and $R\left(D^{*}\right)$ [18], which shows $3.9 \sigma$

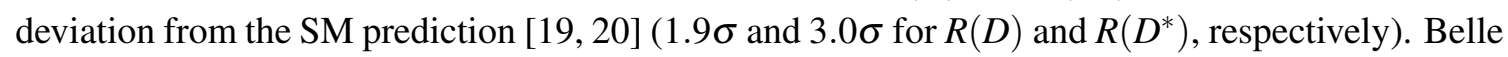
updates the $R\left(D^{*}\right)$ measurement with the semileptonic tagging method [21], which is the first time that the semileptonic tagging is applied to the $\bar{B} \rightarrow D^{(*)} \tau^{-} \bar{v}_{\tau}$ measurement. Since a larger amount of background than that of the hadronic tagging is predicted due to less kinematic constraints. the experimentally cleanest decay mode of $\bar{B}^{0} \rightarrow D^{*+} \tau^{-} \bar{v}_{\tau}$ followed by $\tau^{-} \rightarrow \ell^{-} \bar{v}_{\ell} v_{\tau}$ is selected. The final state is common between the $\bar{B}^{0} \rightarrow D^{*+} \tau^{-}\left(\rightarrow \ell^{-} \bar{v}_{\ell} v_{\tau}\right) \bar{v}_{\tau}$ and the tagging mode of the decay of $\bar{B}^{0} \rightarrow D^{*+} \ell^{-} \bar{v}_{\ell}$ : a $D^{*+}$, a $\ell^{-}$and a missing momentum. In order to separate $\bar{B}^{0} \rightarrow D^{*+} \tau^{-} \bar{v}_{\tau}$ events (signal) from $\bar{B}^{0} \rightarrow D^{*+} \ell^{-} \bar{v}_{\ell}$ events (normalization), a feature that the signal mode has two more neutrinos than the normalization mode is used; $M_{\text {miss }}^{2}$ is larger than 0 , a summed energy used for the event reconstruction is smaller, and a $\cos \theta_{B-D^{(*)} \ell}$ takes negative value in the case of signal events. These variables are analyzed in a multivariate algorithm based on the NeuroBayes package [3].

An extended maximum likelihood fit to the NeuroBayes output distribution and the $E_{\mathrm{ECL}}$ distribution is performed with two-dimensional histogram probability density functions (PDFs). Here, $E_{E C L}$ denotes a summed energy on the electromagnetic calorimeter not used for the event reconstruction. This variable helps to separate signal and normalization events from other background events, such as $\bar{B} \rightarrow D^{* *} \ell^{-} \bar{v}_{\ell}$ and events containing falsely-reconstructed $D^{(*)}$ candidates. These background events often have unreconstructed $\pi^{0}$ and therefore make $E_{\mathrm{ECL}}$ distributions flat. The preliminary result of the $R\left(D^{*}\right)$ measurement with the semileptonic tagging is

$$
R\left(D^{*}\right)=0.302 \pm 0.030 \text { (stat.) } \pm 0.011 \text { (syst.) }
$$

consistency of which with the SM prediction is $1.6 \sigma$. Main sources of the systematic uncertainty are limited statistics of Monte Carlo (MC) simulation samples for construction of the PDFs, an uncertainty of the $\bar{B} \rightarrow D^{* *} \ell^{-} \bar{v}_{\ell}$ modeling which causes uncertainty on the PDF shape, etc. By including the new preliminary result, the HFAG reports that the deviation of $R\left(D^{*}\right)$ from the SM prediction slightly increases to $3.3 \sigma$, and the combination of discrepancies of $R(D)$ and $R\left(D^{*}\right)$ is $4.0 \sigma$ (Fig 2). 


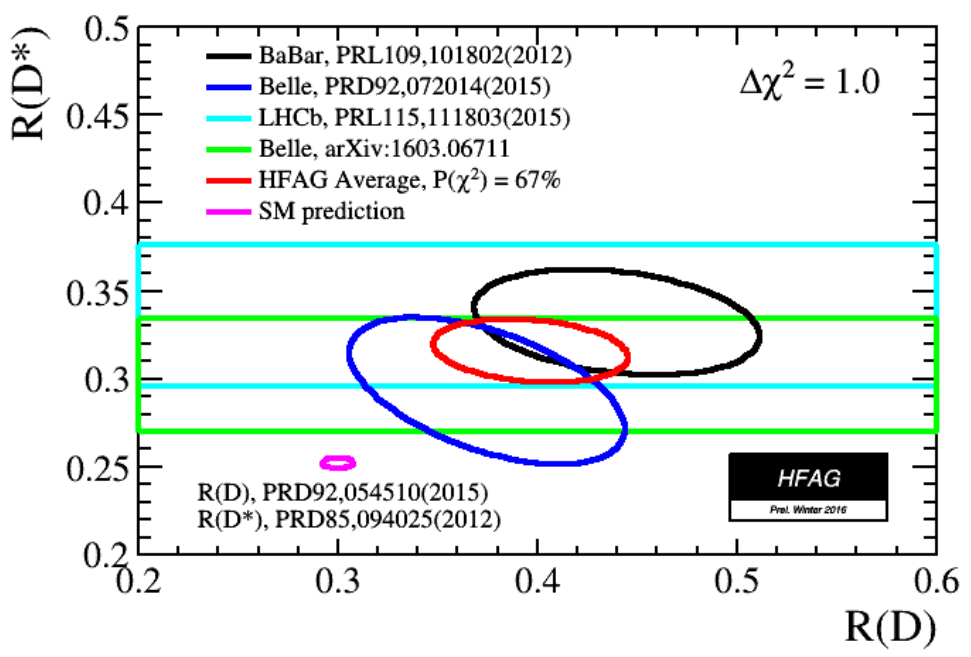

Figure 2: $R(D)$ and $R\left(D^{*}\right)$ average with the latest four results by HFAG [18].

NP contributions to the decay $\bar{B} \rightarrow D^{(*)} \tau^{-} \bar{v}_{\tau}$ are theoretically studied with the model-independent effective Lagrangean [22]

$$
-\mathscr{L}=2 \sqrt{2} G_{F} V_{c b}\left(\delta_{\tau} \mathscr{O}_{V_{1}}+\sum_{i=V_{1}, V_{2}, S_{1}, S_{2}, T} C_{i} \mathscr{O}_{i}\right),
$$

where $\mathscr{O}_{i}$ and $C_{i}$ are operators of 4-fermion couplings and corresponding Wilson coefficients of NP contributions with the subscript $i: V_{1}$ and $V_{2}$ for the vector currents, $S_{1}$ and $S_{2}$ for the scalar currents, and $T$ for the tensor current. Definition of each operator is found in Ref. [22]. The coefficient $\delta_{\tau}$ represents the SM contribution.

Belle tests two NP models with momentum distributions of $D^{*}$ and $\ell^{-}$as well as $R\left(D^{*}\right)$. An signal reconstruction efficiency is corrected as a function of the theoretical parameters, which is estimated using MC simulation. The first model is the type-II 2HDM, where BaBar has reported on their result in Ref. [16] that they excluded this model at a $99.8 \%$ confidence level. In this model, the scalar-type Wilson coefficients have real values:

$$
\begin{aligned}
C_{S_{1}} & =-\frac{m_{b} m_{\tau}}{m_{H^{+}}^{2}} \tan ^{2} \beta, \\
C_{S_{2}} & =-\frac{m_{c} m_{\tau}}{m_{H^{+}}^{2}} .
\end{aligned}
$$

Here, $m_{b}$ and $m_{c}$ are masses of the $b$ quark and the $c$ quark, respectively. A parameter of the type-II $2 \mathrm{HDM}$ is $\tan \beta / m_{H^{+}}$, where $\tan \beta$ is a ratio of the vacuum expectation values of two Higgs doublets and $m_{H^{+}}$is the charged Higgs mass. The charged Higgs mass must be larger than $480 \mathrm{GeV} / c^{2}$ due to a constraint by $b \rightarrow s \gamma$ studies [23] and therefore sufficiently heavy compared to the $c$ quark. Under this assumption, the $C_{S_{2}}$ vanishes and only $C_{S_{1}}$ contributes to the decay in most of the phase space. The second model is the $R_{2}$-type leptoquark model [24], where the scalar and the tensor currents appear with the relation $C_{S_{2}}=7.8 C_{T}$. Although the Wilson coefficients may take complex values, only real numbers are assumed in the test. 


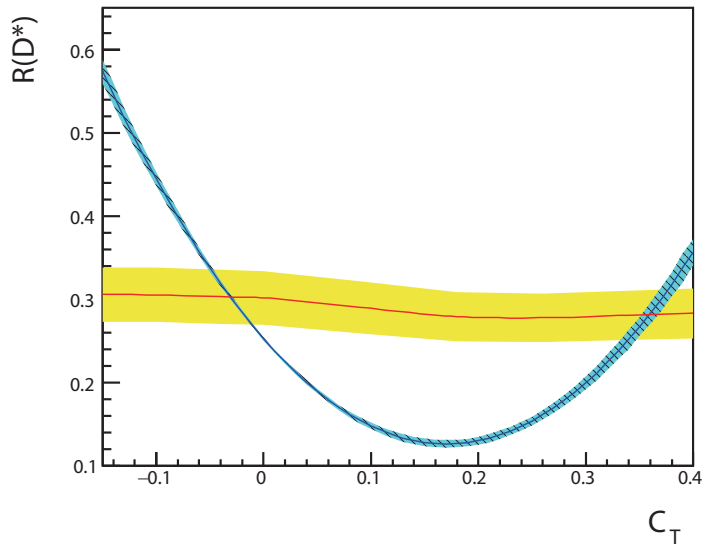

(a) $R\left(D^{*}\right)$ as a function of $C_{T}$.

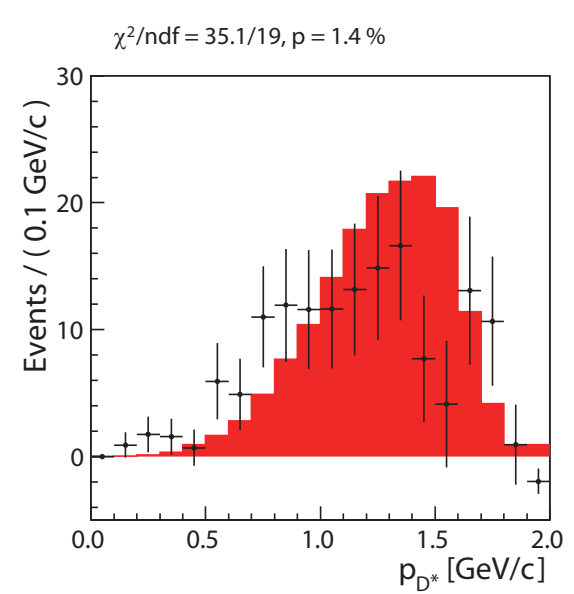

(b) $D^{*}$ momentum distribution at $C_{T}=$ +0.36 .

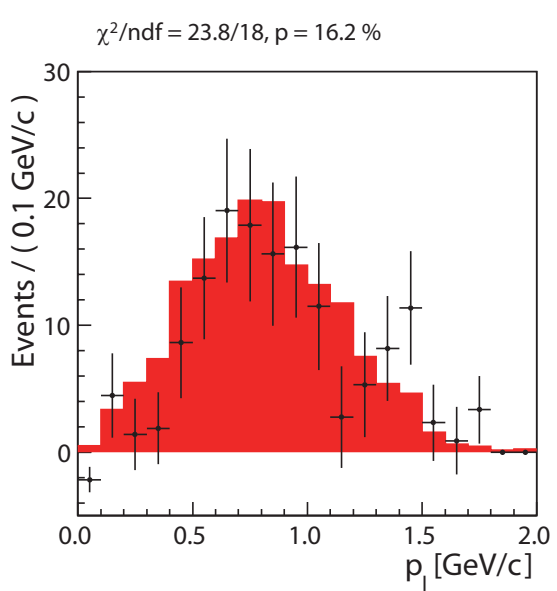

(c) $\ell^{-}$momentum distribution at $C_{T}=$ +0.36 .

Figure 3: (a) $R\left(D^{*}\right)$ result for the $R_{2}$-type leptoquark model; the experimental result is shown by the yellow region $(1 \sigma)$ with its center value by orange curve and the theoretical prediction with $1 \sigma$ uncertainty by the shaded region. (b) and (c) are momentum distributions of $D^{*}$ and $\ell^{-}$, respectively. Background-subtracted data and MC distributions are compared as the points and the solid red histogram, respectively. These figures are cited from Ref. [21].

Figure 3 (a) shows the measured $R\left(D^{*}\right)$ as a function of $C_{T}$ for the $R_{2}$-type leptoquark model. The result has two favored region: around $C_{T}=-0.03$ (SM-like) and around $C_{T}=+0.36$ (NPlike). The corresponding $D^{*}$ and $\ell^{-}$momentum distributions at the NP-like point are shown in Fig. 3 (b) and (c). These momentum distributions disfavor the NP-like point with $p$-values of $1.4 \%$ $\left(D^{*}\right)$ and $16.2 \%\left(\ell^{-}\right)$, respectively. A favored $\tan \beta / m_{H^{+}}$region around 0.7 and compatibility of the $D^{*}$ and $\ell^{-}$momentum distributions are also reported for the type-II 2HDM. 
There are two other $\bar{B} \rightarrow D^{*} \tau^{-} \bar{v}_{\tau}$ analyses ongoing at Belle: the one with the hadronic tagging and the hadronic $\tau$ decays of $\tau^{-} \rightarrow \pi^{-} v_{\tau}$ and $\tau^{-} \rightarrow \rho^{-} v_{\tau}$, and the other with the inclusive tagging, the $\tau$ decays of $\tau^{-} \rightarrow \ell^{-} \bar{v}_{\ell} v_{\tau}$ and $\tau^{-} \rightarrow \pi^{-} v_{\tau}$. The inclusive tagging is one of the $B_{\text {tag }}$ fullreconstruction techniques without specifying exclusive $B$ decay chains [12, 13]. A $D^{*}$ and a $\ell^{-}$ are firstly reconstructed in an event, and remaining particles are collected to form a $B_{\text {tag }}$ candidate. These ongoing analyses will provide with polarization information of $\tau$ and $D^{*}$ as well as $R\left(D^{*}\right)$, which are additional probes to NP [22].

\section{2 $\bar{B}^{0} \rightarrow \pi^{+} \tau^{-} \bar{v}_{\tau}$ with hadronic tagging at Belle}

An exclusive semitauonic decay of $\bar{B}^{0} \rightarrow \pi^{+} \tau^{-} \bar{v}_{\tau}$ contains $b \rightarrow u$ transition. Although its branching fraction is suppressed by a factor of $\left|V_{u b} / V_{c b}\right|^{2} \sim \mathscr{O}\left(10^{-2}\right)$ compared to $\bar{B} \rightarrow D^{(*)} \tau^{-} \bar{v}_{\tau}$, this decay mode may give a hint of the $4.0 \sigma R\left(D^{(*)}\right)$ discrepancy between the experimental result and the SM prediction. The branching fraction is predicted to be $\mathscr{B}\left(\bar{B}^{0} \rightarrow \pi^{+} \tau^{-} \bar{v}_{\tau}\right)=(9.35 \pm$ $0.38) \times 10^{-5}$ [25], which may be enhanced or diminished by existence of NP [26].

Belle searches the decay of $\bar{B}^{0} \rightarrow \pi^{+} \tau^{-} \bar{v}_{\tau}$ with the hadronic tagging [27]. Here, the $\tau$ decays of $\tau^{-} \rightarrow e^{-} \bar{v}_{e} v_{\tau}, \pi^{-} v_{\tau}$ and $\rho^{-} v_{\tau}$ are used. The $\tau^{-} \rightarrow \mu^{-} v_{\mu} v_{\tau}$ channel is not included in the signal extraction due to the low reconstruction efficiency of low momentum muons. However, this mode is used as a veto mode; Events reconstructed as the $\tau^{-} \rightarrow \mu^{-} \bar{v}_{\mu} v_{\tau}$ mode are rejected.

After selection of a $B_{\text {tag }}$ candidate and a pair of opportitely-charged tracks (and one additional $\pi^{0}$ for the $\tau^{-} \rightarrow \rho^{-} v_{\tau}$ mode), signal events are extracted with $E_{\mathrm{ECL}}$ distributions. As a result, a significance of $2.4 \sigma$ is obtained, which is consistent with a $95 \%$ confidence level upper limit of $\mathscr{B}\left(\bar{B}^{0} \rightarrow \pi^{+} \tau^{-} \bar{v}_{\tau}\right)<2.8 \times 10^{-4}$. As this upper limit reaches by a factor of 3 above the SM prediction, the $\bar{B}^{0} \rightarrow \pi^{+} \tau^{-} \bar{v}_{\tau}$ measurement is an interesting subject at Belle II.

\section{Summary}

Four recent topics related to semileptonic $B$ decays from Belle and BaBar were reviewed, which have been published or presented in the past year. In the $\bar{B} \rightarrow D \ell^{-} \bar{v}_{\ell}$ measurement at Belle, the $\left|V_{c b}\right|$ is extracted with two different form factor parametrization. It resulted in $\left|V_{c b}\right|=$ $(40.12 \pm 1.34) \times 10^{-3}$ with the CLN parametrization and $\left|V_{c b}\right|=(41.10 \pm 1.14) \times 10^{-3}$ with the BGL parametrization, which are consistent with both of the averages of inclusive and exclusive measurements. BaBar measured the charmed-semileptonic $B$ decays with two associated pions. They reported the first observation of $\bar{B} \rightarrow D^{0} \pi \pi \ell \bar{v}_{\ell}$ with $5.0 \sigma$ and the first evidence of $\bar{B} \rightarrow D^{+} \pi \pi \ell \bar{v}_{\ell}$ and $\bar{B} \rightarrow D^{*+} \pi \pi \ell \bar{v}_{\ell}$ with $3.0 \sigma$. Belle updated the $R\left(D^{*}\right)$ measurement for the semitauonic decays of $\bar{B} \rightarrow D^{*} \tau^{-} \bar{v}_{\tau}$ with the semileptonic tagging, which was the first measurement of $R\left(D^{*}\right)$ with this method. The measurement resulted in $R\left(D^{*}\right)=0.302 \pm 0.030$ (stat.) \pm 0.011 (syst.) (preliminary), which slightly enlarged the discrepancy of $R\left(D^{*}\right)$ between the average of the experimental results and the SM prediction from $3.0 \sigma$ to $3.3 \sigma$. The combination of $R(D)$ and $R\left(D^{*}\right)$ became $4.0 \sigma$ including this result. Belle is further proceeding additional $\bar{B} \rightarrow D^{*} \tau^{-} \bar{v}_{\tau}$ analyses, which includes polarization measurements of $\tau$ and $D^{*}$. The unmeasured semitauonic decay $\bar{B}^{0} \rightarrow \pi^{+} \tau^{-} \bar{v}_{\tau}$ was searched by Belle, which resulted in the $95 \%$ confidence level upper limit of $\mathscr{B}\left(\bar{B}^{0} \rightarrow \pi^{+} \tau^{-} \bar{v}_{\tau}\right)<2.8 \times 10^{-4}$. This is by a factor of 3 above the SM prediction. 
Although more than six years have passed since shut-down of two $B$ Factory experiments, both Belle and $\mathrm{BaBar}$ collaborations are active for semileptonic $B$ decay analyses. In addition, the LHCb collaboration at the large hadron collider is also capable of searches of specific semileptonic $B$ decays [28]. New results will be provided further in near future, which will be important steps for the Belle II experiment (2017-) and the LHCb Run-3 (2021-).

\section{References}

[1] For example, J.F. Gunion, et. al, Front.Phys. 80 (2000), 1-404 (1989).

[2] M. Feindt, et al., Nucl. Instr. and Meth. A 654 (2011) 432.

[3] M. Feindt, arXiv:physics/0402093 (2004).

[4] For example, Belle and BaBar Collaborations, Eur. Phys. Jour. C 74 (2014) 3026 Section 7.4.1.1.

[5] B. Aubert et al. (BaBar Collaboration), Phys. Rev. D 81, 051101(R) (2010).

[6] K. Hara et al. (Belle Collaboration), Phys. Rev. D 82, 071101(R) (2010).

[7] R. Glattauer et al. (Belle Collaboration), Phys. Rev. D 93, 032006 (2016).

[8] K. Abe et al. (Belle Collaboration), Phys. Lett. B 526, 258 (2002).

[9] I. Caprini, L. Lellouch and M. Neubert, Nucl. Phys. B 530, 153 (1998).

[10] C.G. Boyd, B. Grinstein and R.F. Lebed, Phys. Rev. Lett. 74, 4603 (1995).

[11] F.U. Bernlochner, Z. Ligeti and S. Turczyk, Phys. Rev. D 85, 094033 (2012).

[12] A. Matyja et al. (Belle Collaboration), Phys. Rev. Lett. 99, 191807 (2007).

[13] A. Bozek et al. (Belle Collaboration), Phys. Rev. D 82, 072005 (2010).

[14] M. Huschle et al. (Belle Collaboration), Phys. Rev. D 92, 072014 (2015).

[15] B. Aubert et al. (Belle Collaboration), Phys. Rev. Lett. 100, 021801 (2008).

[16] J.P. Lees et al. (BaBar Collaboration), Phys. Rev. Lett. 109, 101802 (2012).

[17] R. Aaij et al. (LHCb Collaboration), Phys. Rev. Lett. 115, 111803 (2015).

[18] Y. Amhis et al. (Heavy Flavor Averaging Group), arXiv:1412.7515 (2014) and online update at http://www.slac.stanford.edu/xorg/hfag/.

[19] H.Na. et al. (HPQCD Collaboration), Phys. Rev. D 92, 054510 (2015).

[20] S. Fajfer, J.F. Kamenik and F. Mescia, Phys. Rev. D 85, 094025 (2012).

[21] A. Abdesselam et. al (Belle Collaboration), arXiv:1603.06711 (2016).

[22] M. Tanaka and R. Watanabe, Phys. Rev. D 87, 034028 (2013).

[23] M. Misiak et. al, Phys. Rev. Lett. 114, 221801 (2015).

[24] Y. Sakaki, R. Watanabe, M. Tanaka and A. Tayduganov, Phys. Rev. D 88, 094012 (2013).

[25] This estimate is based on private communication by the author of Ref. [27].

[26] For example, R. Dutta, A. Bhol and A.K. Giri, Phys. Rev. D 88, 114023 (2013).

[27] P. Hamer et al. (Belle Collaboration), Phys. Rev. D 93, 032007 (2016).

[28] The LHCb Collaboration, Nature Physics 11, 743-747 (2015). 